\title{
Der Schutz unseres Lebensraums
}

\section{Der physikalisch-chemische Aspekt:}

\section{Kontrolle der anthropogenen Stoffflüsse}

In vorindustrieller Zeit, ja zum Teil auch noch im 19. und in der ersten Hälfte des 20. Jahrhunderts, glaubte man nicht ohne Grund, Abfälle ohne direkte Wiederverwertungseignung vertrauensvoll der Natur überlassen zu können. Der anthropogene Eintrag blieb, wenn man absah von hygienisch fragwürdigen Schadstoffkonzentrationen im lokalen Bereich, bedeutungslos im Vergleich zu den intakten natürlichen Stoffflüssen. Inzwischen hat sich die Situation in doppelter Hinsicht verschlechtert: Die Flut der Schadstoffe, die der Mensch in das Wasser, in die Luft und in den Boden gelangen läßt, ist dramatisch gestiegen, und gleichzeitig hat darin der Anteil an nicht oder schwer abbaubaren sowie an toxischen Komponenten in besorgniserregendem $\mathrm{Maß}$ zugenommen. Allmählich wächst die Einsicht, daß wir damit längerfristig unsere eigenen Lebensgrundlagen oder jedenfalls jene unserer Nachkommen gefährden.

Wie dem zu begegnen wäre, liegt auf der Hand: Es geht darum, das Anschwellen des anthropogenen Stoffflusses in Grenzen zu halten, diesen soweit nötig zu reduzieren und daraus Substanzen, die sich in bezug auf die Rückführung in die Kreisläufe der Natur als problematisch erweisen, fernzuhalten. Wir müssen wegkommen von Einwegprozessen, die in irgendwelchen Senken enden, wegkommen auch vom linearen Denken, das solchem Verhalten zu Grunde liegt; und wir müssen Konsequenzen ziehen aus der Erkenntnis, daß nur von erschöpfbaren Energiequellen unabhängige zyklische Prozesse Gewähr bieten für eine dauerhafte Entwicklung.

Der Schlüssel zur Bewältigung der ökologischen Problematik ist eine nachhaltige Lösung der Energiefrage. Konkret geht es um den Abbau unserer gefährlichen Abhängigkeit von den fossilen Energieträgern. Davon sind wir, wie der weitere Anstieg des Weltkonsums zeigt, noch gefährlich weit entfernt. Vorläufig nutzen wir Erdöl, Erdgas und Kohle weiterhin ungehemmt, zu Preisen, welche weder die absehbare Knappheit noch die bei Gewinnung, Verarbeitung, Transport und Gebrauch verursachte Belastung der Biosphäre mit Schadstoffen zum Ausdruck bringen - ganz zu schweigen vom Kli- marisiko. Die Preissignale haben keine Bremswirkung, solange sich das Plündern beschränkter Ressourcen dem Wachstum der Nachfrage anpaßt; vielmehr verleiten sie zu verschwenderischem Verhalten, das, wenn man zum Beispiel an die überbordende Mobilität mit allen Auswirkungen auf die Besiedlung, die Produktionsstrukturen und den weiten Bereich der Freizeitaktivitäten denkt, ökologisch höchst bedenklich ist.

Der zentrale, ja vielleicht der einzige wirklich erfolgversprechende Ansatzpunkt zur Verwirklichung des «Sustainable Development» wäre eine Vervielfachung der Energiepreise, so da $ß$ sie das Aufbrauchen der in Jahrmillionen entstandenen fossilen Energieträger innerhalb von Jahrzehnten oder - im Falle der Kohle - von einigen Jahrhunderten zu bremsen vermöchten. Diese Maßnahme wäre, wie das Ernst U. von Weizsäcker vorschlägt, schrittweise zu verwirklichen, mit einer Steigerungsrate von jährlich beispielsweise sieben Prozent, damit sich die Wirtschaft allmählich an voraussehbare neue Rahmenbedingungen anpassen könnte. Das ist allerdings ein Pfad, der sich nicht für den Alleingang eines Kleinstaates wie der Schweiz eignet. Trotzdem ist es wichtig, daß wir uns im Blick auf internationale Lösungen, ohne die sich die Gefahr einer Ökokatastrophe nicht bannen läßt, rechtzeitig damit befassen.

Man sollte sich allerdings vor Illusionen hüten: Das Ziel läßt sich leicht festlegen auf Grund der Erkenntnis, daß es in einem limitierten System kein grenzenloses, aus dem Verbrauch beschränkter und nicht erneuerbarer Ressourcen alimentiertes Wachstum geben kann: doch wie wir es innerhalb nützlicher Frist erreichen können, bleibt schleierhaft. Noch vermag niemand überzeugend darzulegen, wie sich die Menschheit aus ihrer ungemütlichen Abhängigkeit von den fossilen Brenn- und Treibstoffen befreien soll, die heute zu über 80 Prozent ihren wachsenden Energiebedarf decken. Mit effizienterer Nutzung läßt sich das Verhältnis zwischen nachgefragter Nutzenergie und eingesetzter Primärener-

Walter Schiesser, Redaktor, Neue Zürcher Zeitung, Falkenstraße 11, Postfach, 8021 Zürich 
gie zweifellos noch sehr stark verbessern, doch das ändert nichts daran, da $\beta$ die nutzbaren Vorräte in absehbarer Zukunft knapp werden und daß für das $\mathrm{CO}_{2}$-Problem keine praktikable Lösung in Aussicht steht.

\section{Der biologische Aspekt:}

\section{Erhaltung der Artenvielfalt}

Wie wäre die Situation, wenn dem Menschen mit einem Durchbruch, etwa im Bereiche der Kernfusion, die Erschließung neuer, praktisch unbegrenzter Energiequellen und damit auch die Substitution der fossilen Energieträger gelänge? Wenn zum Beispiel die «kalte Fusion», die vor einiger Zeit Schlagzeilen machte, in absehbarer Zukunft doch noch Wirklichkeit würde? Könnte damit die Wissenschaft die Voraussetzungen schaffen für ein vielleicht energieintensives, aber - wenn man von radioaktiven Abfällen absieht - schadstoffarmes technisches Umwelt-Management? Ließe sich so das Raumschiff Erde mit geschlossenen, unschädlichen Stoffkreisläufen ausstatten? Ist, wie eingeschworene Optimisten, etwa Bruno Fritsch, behaupten, damit zu rechnen, daß der Mensch dank seiner Intelligenz immer noch rechtzeitig einen Ausweg aus ökologischen Engpässen finden wird, ohne daß er sich einzuschränken braucht?

Gegenüber solchen Perspektiven ist Skepsis angebracht: Welche Gewähr wäre dafür geboten, daß mit einem rein technokratischen Ansatz die Lösung der heutigen Probleme - sofern sie überhaupt gelingt - nicht mit noch gravierenderen Problemen erkauft würde? Fest steht jedenfalls, daß die Dominanz des einen Lebewesens Mensch auf diesem Planeten - die eigentliche Ursache der ganzen ökologischen Problematik - weiter zunähme. Damit würde der Verdrängungsprozeß zu Lasten der Artenvielfalt, der in seiner Dramatik heute schon mit dem großen Sterben beim Übergang von der Kreide zum Tertiär vergleichbar ist, tendenziell weiter beschleunigt.

Umweltschützerisches Denken, das auf einem einseitig anthropozentrischen Ansatz beschränkt bliebe, ist a priori unökologisch und greift zu kurz. Es geht dabei um mehr als um den Anspruch von uns Menschen auf uneingeschränkte Lebensqualität, auf eine gewissermaßen als Konsumgut produzierbare "heile» Umwelt. Wir tun uns noch immer schwer mit der Einsicht, daß wir unseren Lebensraum mit anderen, sichtbaren und unsichtbaren, angenehmen und weniger angenehmen Arten teilen und weiterhin teilen müssen - oder vielmehr dürfen. Von «unserem» Lebensraum zu sprechen, wie wir es gewohnt sind, ist deshalb fragwürdig und eigentlich nur angemessen, wenn es aus einem die Gesamtheit der Lebewesen einschließenden «Wir-Ge- fühl» heraus geschieht. Wir sollten also aufhören, die Biosphäre, den dünnen Schleier rund um die Erde, in dem sich das Leben mit seiner noch unbekannten Millionenzahl von Arten ereignet, anthropozentrisch zu vereinnahmen, gnadenlos als Objekt auszubeuten - und genau damit zu zerstören. Das bedingt eine Rückkehr zu einem behutsamen, dialogischen Verhältnis zur Natur, aus dem der Mensch der Aufklärung aufgebrochen war, um sie in den Griff zu nehmen.

\section{Der seelische Aspekt:}

\section{Bewahrung der Kulturlandschaft}

Doch angenommen, es gelänge, nicht nur die Stoffund Energieflüsse mit den Erfordernissen einer nachhaltigen Entwicklung in Einklang zu bringen, sondern auch die Nachfolgeprobleme zu beherrschen und überdies das Artensterben zu bremsen zum Beispiel durch starke Konzentration menschlicher Siedlungsgebiete bei gleichzeitiger Ausscheidung ausgedehnter, anthropogenen Einflüssen teilweise entzogener Kompensationsflächen: Wäre dann alles zum besten bestellt? Kaum. Ein solches Szenario, das die biologische Dimension in das Umwelt-Management einbezieht, mag allenfalls als Theorie attraktiv erscheinen - daß es Wirklichkeit würde, können wir trotzdem im Ernst nicht wünschen, selbst wenn wir dazu in der Lage wären. Denn damit würden wir lediglich der weiteren Entwurzelung des Menschen Vorschub leisten, die schmerzliche Entfremdung von der Natur verstärken und so die Aussichten auf eine Wende in unserem gestörten Verhältnis zur Mitwelt schmälern.

$\mathrm{Da}$ in dieser Beziehung einiges nicht mehr stimmt, läßt sich längst nicht mehr übersehen. $\mathrm{Zu}$ offenkundig ist die wachsende Sehnsucht nach Natur, insbesondere nach Landschaft, die der Mensch noch nicht ruiniert hat. Millionen suchen sie - und beteiligen sich damit kräftig an der raschen Zerstörung der «letzten Paradiese», teils bewußt, teils unbewußt. Daß hier ein gewaltiges ungestilltes Bedürfnis im Spiele ist, erlebt man auf unseren Autobahnen und vor dem "Check-in» der Flughäfen. Dort soll sich der Andrang bis zur Jahrtausendwende verdoppeln, obwohl dieser Luxus für den weitaus größten Teil der Menschheit weiterhin Traum bleiben wird - und auch bleiben muß, weil unser westliches Wohlstandsmodell in einer von der Bevölkerungsexplosion gekennzeichneten Welt nicht exportfähig ist.

Ziel dieser aus dem Unbehagen in der technischen Massenzivilisation geborenen Sehnsüchte ist nicht nur die unberührte Natur in exotischen Weltgegenden; Ziel ist auch die einheimische naturnahe Kulturlandschaft, so wie sie unsere Vorfahren geschaffen haben, im Laufe von Jahrhunderten mühseliger 
Auseinandersetzung mit der so lange als übermächtig empfundenen Natur. Geprägt ist sie von der Landwirtschaft, die im grossen und ganzen bis zum Überhandnehmen einer technisierten, auf fossile Energie abgestützten Nutzung dem Gebot der Nachhaltigkeit genügte. Daß darin die erhaltenen Zeugen menschlicher Aktivität, vor allem Bauwerke, nicht als störend empfunden werden, sondern im Gegenteil als Bereicherung, hängt wohl mit deren zahlenmäßigem Gewicht zusammen und mit der Langsamkeit ihrer Entstehung. Es ist, als habe das zähe Ringen von Generationen, die noch nicht über moderne Technologie und Fremdenergie im Überfluß verfügte, Wertvolleres, Gültigeres hervorgebracht als unsere heutige Wegwerfgesellschaft in ihrem Machtrausch und mit ihrer Zeitraffermentalität. Es könnte sein, daß Dinge so viel wert sind, wie man dafür aufgewendet hat - in diesem Falle nicht an Geld, sondern an Kreativität, Liebe und an Lebenszeit.

$\mathrm{Zu}$ bedenken ist ferner, daß Kulturlandschaft mehr ist als der Schauplatz unserer physischen Existenz. Sie ist der sichtbare, unmittelbar mit den Sinnen erlebbare und damit zutiefst menschliche Teil der Biosphäre. Unsere Wahrnehmung hat sich im Zuge der Evolution so entwickelt, daß wir uns darin $\mathrm{zu}$ behaupten vermochten. Wenn wir nun heute im Begriff stehen, die Landschaft zur Zivilisationssteppe zu degradieren, so führt das nicht nur zur Verarmung der Natur; wir laufen Gefahr, den Boden veröden zu lassen, in dem wir seelisch wurzeln. Ohne innere Beziehung zur Landschaft, in der wir leben, entwickeln wir uns zur Flugsand-Gesellschaft, ohne feste Verankerung, mit einem Mobilitätsverhalten, das sich ökologisch nur negativ auswirken kann. Verschärft wird das hausgemachte Problem durch eine wachsende Migrationsmobilität, die für immer mehr Menschen aus Entwicklungsländern Ursache und zugleich Folge der Entwurzelung in unwirtlich gewordener Umwelt ist. Wir stecken hier in einem Teufelskreis: Mit der Natur den Frieden zu schließen, dessen sie und wir so dringend bedürfen, wird nicht einfacher, wenn es immer mehr Heimatlose gibt, die kaum mehr eine positive emotionale Bindung an die Erde haben können, die sie trägt.

Bewahrung der Kulturlandschaft: Dazu bedarf es nicht in erster Linie einer immer größeren Fülle von Maßnahmen, deren Wirkung meistens darauf beschränkt bleibt, die schädlichen Auswirkungen im Verhältnis zum zivilisatorischen Nutzen im herkömmlichen Sinne zu minimieren. Weit wichtiger wäre es, Ausmaß und Tempo der Veränderung zu verringern, um eine dauerhafte Entwicklung im Sinne des «Sustainable Development» zu begünstigen, eine Entwicklung, die - abgesehen von den zentralen ökologischen Aspekten - wieder eher menschlichem Maß entsprechen würde. Daß das vermutlich ein frommer Wunsch bleiben wird, ändert nichts an der Berechtigung dieser Forderung. Sie ist um so dringlicher, als sich die Kräfte, mit denen der Mensch den Reichtum der Natur zerstört, zuletzt gegen ihn selber richten werden - gegen seine physischen Lebensgrundlagen ebenso wie gegen seine seelischen. Der Ruf nach Bewahrung und behutsamer Weiterentwicklung unserer Kulturlandschaft ist somit untrennbar verbunden mit der Notwendigkeit, die Voraussetzungen für eine dauerhafte Entwicklung zu schaffen. 